\title{
Correspondence
}

\section{Rescue from early-onset hearing loss in a mouse model lacking the cyclin-dependent kinase inhibitor p19 1 Ink4d}

\author{
$Q \mathrm{Ma}^{1,4}, \mathrm{M} \mathrm{Grati}{ }^{1,4}, \mathrm{~F} \mathrm{Bai}{ }^{2,4}, \mathrm{JPei}^{1}, \mathrm{X}-\mathrm{H} \mathrm{Pei}{ }^{2}$ and X Liu ${ }^{\star, 1,3}$ \\ Cell Death and Disease (2016) 7, e2131; doi:10.1038/cddis.2016.38; published online 10 March 2016
}

\section{Dear Editor}

Human and mouse genetic-based approaches have been instrumental in identifying key genes and deciphering mechanisms essential for the development, maturation, function and maintenance of auditory organ. In particular, the absence of mammalian inner ear sensory hair cell $(\mathrm{HC})$ regenerative capabilities drove researchers towards gathering clues on their differentiation and maintenance; this would help in the future design of approaches for enhancing HCs' maintenance power and eventually inducing their regeneration. ${ }^{1}$ Mechanisms responsible for cell cycle exit, epithelium patterning and post-mitotic cell cycle arrest of maturing and mature HCs have been a challenging field of focus in auditory research, and the information so far gathered is rather scarce. ${ }^{2}$ For example, in a mouse line lacking cyclin-dependent kinase inhibitor $p 19^{\operatorname{lnk} 4 d}(p 19)$, Chen et al. ${ }^{3}$ have shown that $p 19$ does not influence mouse embryonic stereotyped patterning of cochlea zone of nonproliferating cells into the organ of Corti that is composed of a mosaic of sensory HCs and supporting cells occurring around embryonic days 15.5 and $17.5 .^{3}$ They reported, however, a late progressive loss of sensory $\mathrm{HCs}$ accompanied by progressive hearing loss (HL) starting at 2.5 weeks postnatal. We initiated further studies into p19 network regulating $\mathrm{HC}$ survival. We detected the presence of p19 starting at postnatal day 3 (P3) and thereafter in cochlea outer (OHCs) and inner HCs (IHCs; Figure 1a). We also generated a new mouse p19 knockout line $\left(p 19^{-/}\right)$, where $p 19$ has been entirely deleted. ${ }^{4}$ On these mice, we performed auditory brainstem recordings (ABRs) at P23 that showed a severe to profound $\mathrm{HL}$ (Figure 1b). We also used whole-mount immunofluorescence to assess at this stage HC's survival and stereocilium hair bundle morphology, and observed massive death and degeneration of OHCs that are known for their increased vulnerability, and to a lower extent the death of IHCs, accompanied by a generalized severe disorganization of remaining $\mathrm{HC}$ stereocilium hair bundles (Figure 1c, P23, green). We looked at the chronology of the $\mathrm{HC}$ death and degeneration events, and found that very sporadic $\mathrm{HC}$ death at the mid-basal and basal regions of the cochlea started as early as P7 (Figure 1c at P3 and P7, green). Then, $\mathrm{HC}$ death has rapidly spread towards the cochlea apex and increased exponentially along all cochlea turns at P12 and thereafter (Figure 1c, P12 and P23, green). However, no vestibular $\mathrm{HC}$ death was detectable in $p 19^{-1-}$ mice (not shown). p19, one of the Ink4 family of proteins, exclusively bind to and inhibit cyclin-dependent kinases Cdk4 and Cdk6. ${ }^{5}$ To test the hypothesis if $\mathrm{HC}$ death in $p 19^{-/-}$mice is due to uncontrolled activity of Cdk4, we created double knockout mice $p 19^{-/} ; \mathrm{Cdk}^{-/-5,6}$ Indeed, hearing had been perfectly recovered in these mice and $A B R$ thresholds were very comparable at all three tested frequencies to agematched wild-type mice (Figure $1 \mathrm{~b}$ ) and no HC loss was detectable by immunofluorescence (Figure 1c at P12 and $\mathrm{P} 23$, yellow). To our knowledge, this is the first study demonstrating rescuing role of Cdk4 deletion for p19 deficiency in vivo. Given the complexity of cell cycle control in mammals, the rescue of 19 loss-induced $\mathrm{HC}$ death and $\mathrm{HL}$ by Cdk4 deletion is surprising and demonstrates conclusively the antagonizing functional regulation of Cdk4 and p19 in post-mitotic maintenance in vivo. Inappropriate cell cycle reentry in response to defects in cell cycle control pathways may be a general mechanism through which sensory HCs are damaged or lost. These results further indicate that inhibition of Cdk4 activity by using drugs, such as PD 0332991 that is an FDA approved agent for cancer treatment, could have potential to treat deaf patients caused by aberrant cell cycle re-entry.

\footnotetext{
${ }^{1}$ Department of Otolaryngology (D-48), University of Miami Miller School of Medicine, Miami, FL, USA; ${ }^{2}$ Molecular Oncology Program, Department of Surgery, University of Miami Miller School of Medicine, Miami, FL, USA and ${ }^{3}$ Department of Otolaryngology, Xiangya Hospital, Central South University, Changsha, China ${ }^{*}$ Corresponding author: X Liu, Department of Otolaryngology (D-48), University of Miami Miller School of Medicine, 1666 NW 12th Avenue, Miami, FL 33136, USA. Tel: 305 243 1484; Fax: 305243 2009; E-mail: xliu@med.miami.edu

${ }^{4}$ These authors contributed equally to this work.
} 
a

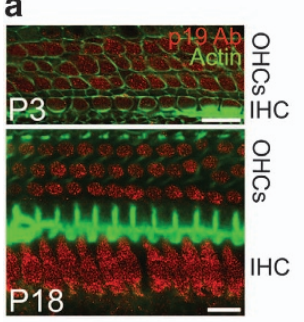

b

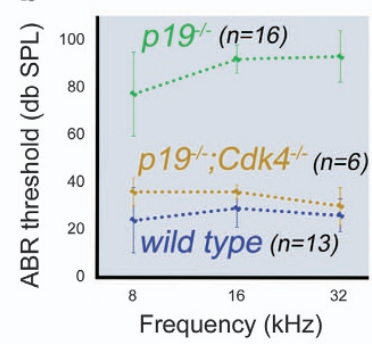

C

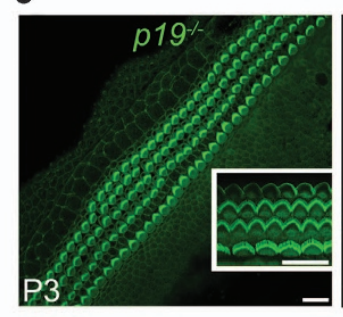

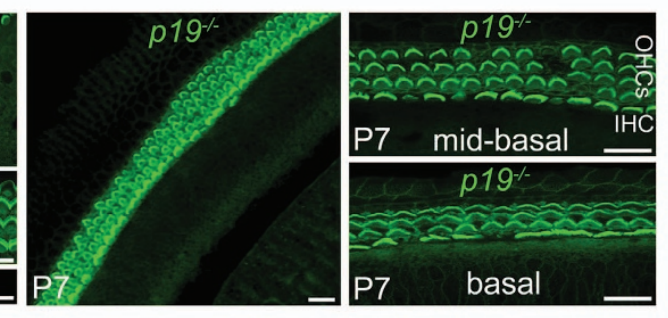

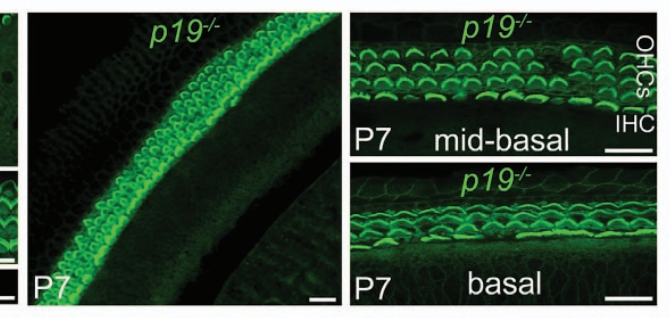

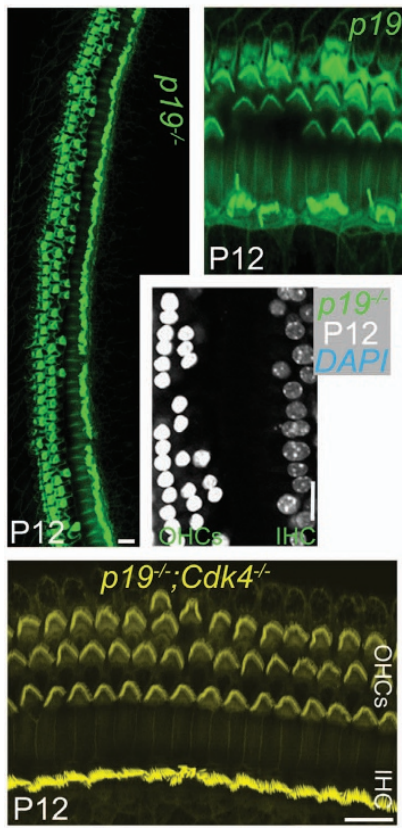

\section{-}
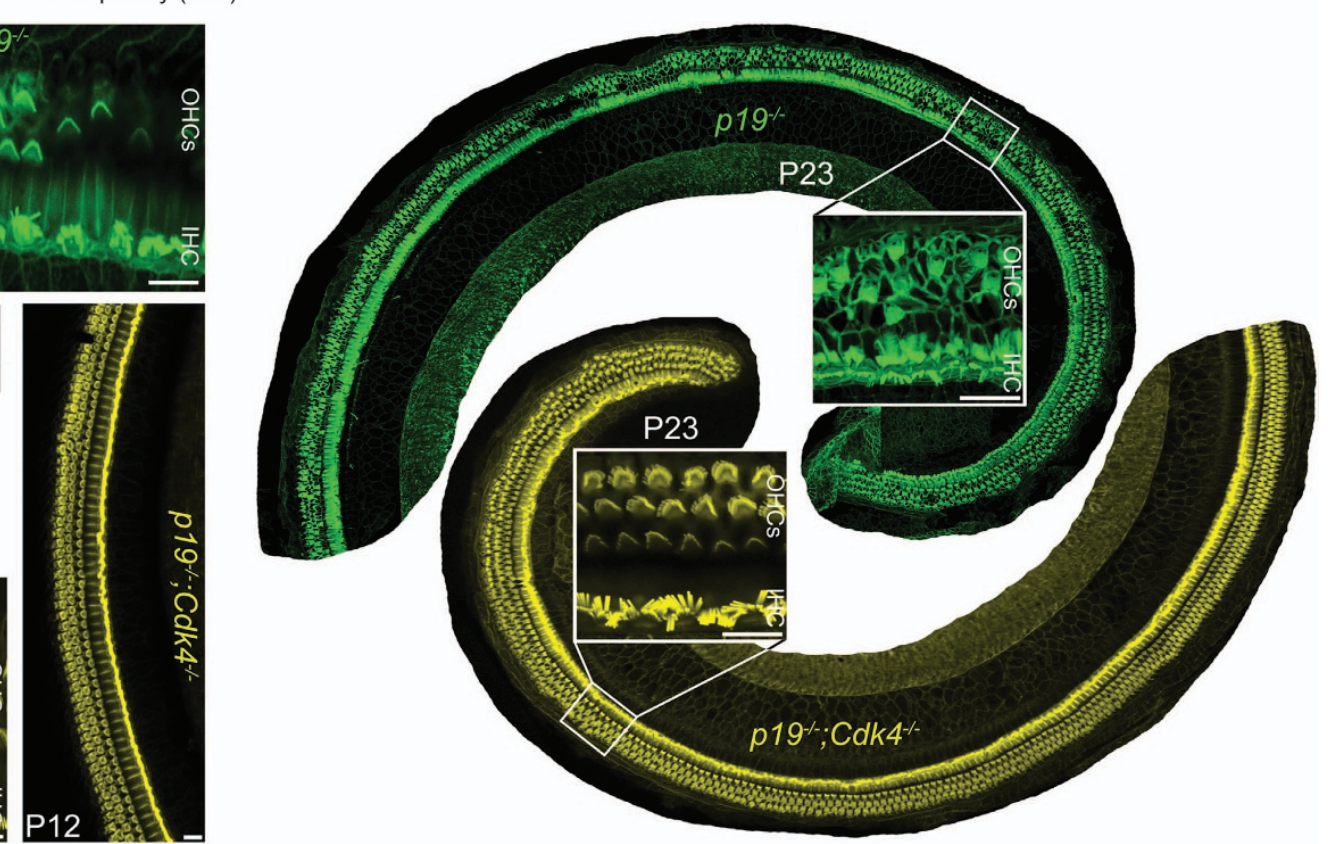

Figure 1 Cdk4 deletion in p19 $9^{-/}$mice rescues hair cell (HC) survival and restores auditory function. (a) Whole-mount immunofluorescence of postnatal day 3 (P3) and 18 (P18) mouse cochlea using rabbit polyclonal antibody (sc-1063; Santa Cruz Biotechnology, Dallas, TX, USA) showing the early detection of p19 (red) at P3, and thereafter at P18 in all three rows of outer $(\mathrm{OHCs})$ and one row of inner sensory $\mathrm{HCs}(\mathrm{IHC})$; actin is counterstained with phalloidin in green. Immunofluorescence experiments were performed as described. ${ }^{7}$ (b) Average thresholds of auditory brainstem recordings (ABRs) at pure tone frequencies 8,16 and $32 \mathrm{kHz}$ on P23-day-old $p 19^{-/-}$(green; $n=16$ ) mice and their $p 19^{-1-} ; C d k 4^{-/-}$double knockout (brown; $n=6$ ) littermates, as well as their age-matched wild-type mice (blue; $n=13$ ) showing high thresholds at all frequencies in $p 19^{-/-}$mice synonymous of a severe to profound hearing loss, and much lower thresholds for $p 19^{-/} ; C d k 4^{-1}$ mice comparable to normal hearing wild-type mice. ABRs were performed as described. ${ }^{8}$ (c) Representative low- and high-magnification confocal micrographs of whole-mount immunofluorescence preparations of P3, P7, P12 and P23 cochlea from p19 ${ }^{-1-}$ mice (green), and of P12 and P23 cochlea from $p 19^{-1-} ; C d k 4^{-/-}$mice (yellow). All preparations were stained with phalloidin to reveal actin, with the exception of one DAPI-stained

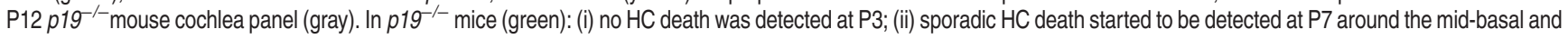
basal turn of the cochlea; (iii) $\mathrm{HC}$ death and degeneration, and stereocilium bundle disorganization increased significantly at P12; and (iv) massive death of OHCs and IHCs, and damage of remaining $\mathrm{HC}$ stereocilium bundles is observable at P23. In $\mathrm{p}^{19^{-/-}} ; \mathrm{Cdk}^{-/-}$mice (yellow), complete rows of OHCs and IHCs, and normal HC stereocilium bundles have been seen at all stages. Scale bars, $20 \mu \mathrm{m}$

\section{Conflict of Interest}

The authors declare no conflict of interest.

Acknowledgements. This work was supported by R01 DC05575, R01 DC01246 and R01 DC012115 from the National Institutes of Health/National Institute on Deafness and Other Communication Disorders to (XL), a DOD Idea Award (W81XWH-10-1-0302) and startup funds from the University of Miami Miller School of Medicine to (XHP).

1. Kopecky B, Fritzsch B. Cells 2012; 1: 667-698.

2. Baumgartner B, Harper JW. Nat Cell Biol 2003; 5: 385-387.

3. Chen P et al. Nat Cell Biol 2003; 5: 422-426.

4. Bai F et al. Mol Cell Biol 2014; 34: 2121-2134.

5. Pei XH, Xiong Y. Oncogene 2005; 24: 2787-2795.
6. Tsutsui T et al. Mol Cell Biol 1999; 19: 7011-7019.

7. Grati M, Kachar B. Proc Natl Acad Sci USA 2011; 108: 11476-11481.

8. Noben-Trauth K et al. PLoS One 2010; 5: e11459.

(i) Cell Death and Disease is an open-access journal published by Nature Publishing Group. This work is licensed under a Creative Commons Attribution 4.0 International License. The images or other third party material in this article are included in the article's Creative Commons license, unless indicated otherwise in the credit line; if the material is not included under the Creative Commons license, users will need to obtain permission from the license holder to reproduce the material. To view a copy of this license, visit http://creativecommons.org/licenses/by/4.0/ 